\title{
Polyradiculonévrite aiguë et glomérulonéphrite extramembraneuse au décours d'une primo-infection à Epstein-Barr virus chez une patiente de 12 ans
}

\author{
Acute inflammatory polyradiculoneuropathy and membranous \\ glomerulonephritis following Epbstein-Barr virus primary \\ infection in a 12-year-old girl \\ P. Meyer ${ }^{a}$, S. Soëte ${ }^{a}$, P. Raynaud ${ }^{b}$, V. Henry ${ }^{a}$, D. Morin' ${ }^{c}$, M. Rodière ${ }^{d}$, F. Rivier $^{a}$, \\ A. Roubertie $e^{a, *, e}$ \\ a Service de neuropédiatrie, hôpital Gui-de-Chauliac, CHU de Montpellier, université \\ Montpellier 1, 80, avenue Fliche, 34295 Montpellier cedex 5, France \\ ${ }^{\mathrm{b}}$ Service d'anatomie pathologique, CHU de Montpellier, université Montpellier 1, 34000 \\ Montpellier, France \\ ${ }^{\mathrm{C}}$ Service de néphropédiatrie, CHU de Montpellier, université Montpellier 1, 34000 Montpellier, \\ France \\ ' Service de maladies infectieuses pédiatriques, CHU de Montpellier, université Montpellier 1, \\ 34000 Montpellier, France \\ Inserm U827, IURC, Montpellier, France
}

\section{Summary}

Acute inflammatory polyradiculoneuropathy, or Guillain-Barré syndrome (GBS), is characterized by peripheral nerve demyelination, which leads to rapidly progressive weakness, loss of sensation, and loss of deep tendon reflexes. It is a prototype of postinfectious autoimmune disease, whose pathophysiology is well described in the forms provoked by certain bacteria (molecular mimicry with Campylobacter jejuni), but remains unclear for the forms related to other organisms (cytomegalovirus, Epstein-Barr virus and other herpes group viruses, Mycoplasma pneumoniae). Glomerular lesions can be associated with the neurological symptoms and have also been described after various infections, independently of any signs of polyradiculoneuropathy. We report the observation of a 12-year-old girl who presented with Guillain-Barré syndrome with facial diplegia, ataxia, and intracranial hypertension following Epstein-Barr virus (EBV) primary infection. During the course of the neurological disease, membranous glomerulonephritis (MGN) was diagnosed. The neurological impairment was regressive within 6 months after intravenous immunoglobulin treatment followed by intravenous then oral corticosteroid administration. Viremia remained high more than 6 months after the onset of symptoms. Glomerulopathy progressed

\section{Résumé}

La polyradiculonévrite aiguë (PRNA) ou syndrome de Guillain-Barré est caractérisée par une démyélinisation des nerfs périphériques entraînant un déficit sensitivomoteur et une aréflexie ostéotendineuse d'évolution rapide. La PRNA est un prototype de maladie autoimmune post-infectieuse, dont la physiopathologie a été bien décrite pour certains germes (mimétisme moléculaire et Campylobacter jejuni) et qui est encore peu connue pour d'autre (cytomegalovirus, Epstein-Barr virus et autres virus du groupe herpès, Mycoplasma pneumoniae). Une atteinte glomérulaire peut être associée à l'atteinte neurologique et a par ailleurs été décrite au cours de certaines infections indépendamment de toute polyradiculonévrite. Nous rapportons l'observation d'une patiente de 12 ans qui a présenté une PRNA avec une diplégie faciale, une ataxie et une hypertension intracrânienne au décours d'une primo-infection à virus Epstein-Barr (EBV) ; puis qui a développé une glomérulonéphrite extramembraneuse (GEM). L'atteinte neurologique a été résolutive en 6 mois après 3 cures d'immunoglobulines intraveineuses suivies de 3 cures de corticoïdes intraveineux avec relais oral. La virémie était restée élevée plus de 6 mois après le début de la symptomatologie. L'atteinte rénale avait évolué pour son propre

\footnotetext{
* Auteur correspondant.

e-mail : a-roubertie@chu-montpellier.fr
} 
independently and finally required immunosuppressant medication with cyclosporine. EBV might be the factor that triggered the autoimmune disorders, as previously reported for systemic lupus erythematosus and multiple sclerosis in children. To the best of our knowledge, this association of 3 conditions (GBS, MGN, and EBV primary infection) has never been reported in the literature.

\section{Introduction}

La polyradiculonévrite aiguë (PRNA) est caractérisée par une démyélinisation des nerfs périphériques entraînant un déficit moteur d'évolution rapide, une aréflexie ostéotendineuse et l'absence ou la pauvreté des symptômes sensitifs. Sa prévalence est estimée entre 0,5 et 1,5/100 ooo enfants dans les pays industrialisés [1]. Une infection précède les symptômes neurologiques dans environ $2 / 3$ des cas [2]. Une atteinte glomérulaire peut être associée à la PRNA comme à certains processus infectieux. Nous rapportons l'observation d'une patiente de 12 ans qui avait développé au décours d'une primo-infection à Epstein-Barr virus (EBV) un tableau de PRNA puis une glomérulonéphrite extramembraneuse (GEM).

\section{Observation}

Cette enfant de 12 ans et demi n'avait pas d'antécédent médicochirurgical particulier. Son développement psychomoteur était normal. Elle avait eu ses premières règles à 11 ans. Elle était la $2^{\mathrm{e}}$ d'une fratrie de 3 enfants et avait pour seuls antécédents familiaux notables une paralysie faciale gauche $a$ frigore chez son père et une polymyosite chez une tante maternelle. L'anamnèse trouvait à l'âge de 11 ans et 11 mois l'apparition de céphalées avec aura visuelle ou sensitive, nausées et vomissements, sans facteur déclenchant identifié, bien améliorées par l'ibuprofene. L'électroencéphalogramme (EEG) était normal. On constatait ensuite une altération de l'état général sans fièvre, avec une asthénie et une perte de poids ayant compromis sa scolarisation.

Quatre mois plus tard, la patiente présentait des myalgies, des difficultés motrices puis une paralysie faciale périphérique gauche secondairement bilatérale (diplégie faciale) associées à des adénopathies diffuses. Le bilan biologique ne montrait qu'une sérologie EBV en faveur d'une primo-infection (immunoglobulines monoclonales de type IgM anti-VCA positives). L'électromyogramme (EMG) enregistrait des signes de compte et avait nécessité un traitement immunosuppresseur par ciclosporine. L'EBV pourrait être le déclencheur de l'auto-immunité dans ce cas, comme cela a déjà été évoqué dans le lupus érythémateux disséminé et la sclérose en plaques chez l'enfant. Nous n'avons pas trouvé d'observation associant les 3 conditions (PRNA, GEM et primo-infection à EBV) dans la littérature française et anglosaxonne.

Mots clés : Epstein-Barr virus, Polyradiculonévrite aiguë, Glomérulonéphrite extramembraneuse

dénervation avec une absence de réponse aux stimulations des nerfs faciaux droit et gauche. Une corticothérapie par voie générale était instaurée sans efficacité.

La patiente nous était adressée pour poursuite de la prise en charge un mois plus tard, à l'âge de 12 ans et 4 mois. L'examen clinique notait une perte de poids de $8 \mathrm{~kg}$ en 4 mois. La patiente était très algique, se plaignant de céphalées, présentant une diplégie faciale persistante, une marche très difficile avec un déficit moteur et une abolition des réflexes ostéotendineux aux membres inférieurs, associés à une ataxie. L'examen du fond de l'œil (FO) montrait un œdème papillaire bilatéral témoin d'une hypertension intracrânienne (HTIC).

La vitesse de sédimentation (VS) était de $50 \mathrm{~mm}$ à la $1^{\text {re }}$ heure. L'hémogramme, le bilan hépatique, l'ionogramme et la fonction rénale étaient normaux. L'analyse du liquide céphalorachidien (LCR) objectivait une dissociation albumino-cytologique avec une hyperprotéinorachie à 2,39 g/l et une cellularité normale. La mesure de pression du LCR était à $32 \mathrm{~cm}$ d'eau. L'EMG était compatible avec une polyradiculonévrite de type démyélinisante (acute inflammatory demyelinating polyneuropathy des anglo-saxons) : un ralentissement des vitesses de conduction et un allongement des latences motrices et sensitives aux 4 membres, des blocs de conduction distal et proximal, et un allongement des latences des ondes $F$. Les potentiels évoqués visuels (PEV) étaient normaux. L'imagerie par résonance magnétique (IRM) cérébromédullaire montrait un rehaussement des méninges de la queue de cheval.

Sur le plan étiologique, la sérologie EBV à l'entrée était toujours en faveur d'une primo-infection récente (IgM antiVCA faiblement positives, index des IgG anti-VCA positif à 2,4 et IgG anti-EBNA négatives). Un bilan infectieux extensif ne montrait qu'une PCR (polymerase chain reaction) positive pour EBV et cytomégalovirus (CMV) dans la moelle osseuse. La PCR EBV dans le LCR était négative. À noter que l'antigénémie EBV n'avait pas été réalisée au début de la prise en charge. Le bilan autoimmun (y compris les anticorps anti-gangliosides) était négatif à plusieurs reprises. Les recherches de sarcoïdose, 


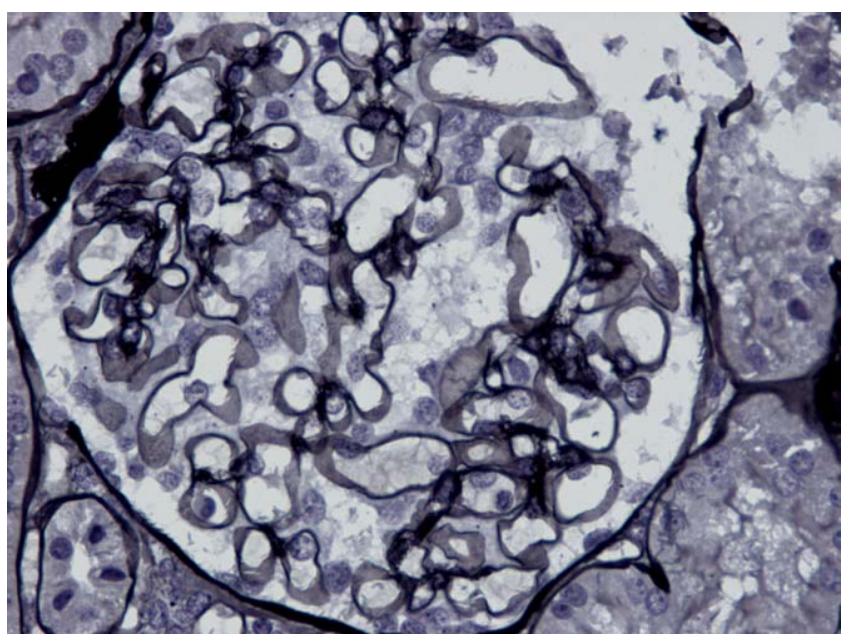

Figure 1. Glomérule de morphologie optique normale : absence d'épaississement des membranes basales glomérulaires (Jones, × 250).

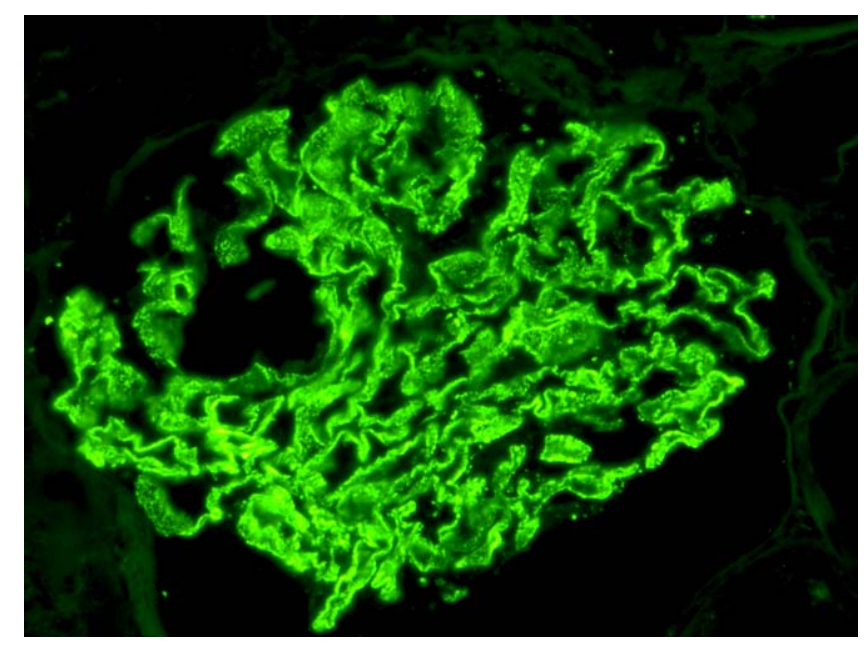

Figure 2. Immunofluorescence : dépôts pariétaux granuleux extramembraneux globaux et diffus (sérum anti-lgG, $\times 250$ ).

de syndrome d'activation macrophagique, de syndromes néoplasique et paranéoplasique étaient négatives.

En raison de ce tableau de PRNA avec HTIC et diplégie faciale, la patiente bénéficiait de 3 cures de veinoglobulines $(2 \mathrm{~g} / \mathrm{kg}$ sur $2 \mathrm{j}$ ) associées à un traitement oral par acétazolamide et de la rééducation motrice. L'amélioration clinique était modeste. La fonction rénale était normale ainsi que l'examen cytologique des urines. Une protéinurie isolée à 1,06 g/24 h apparaissait au décours de la $3^{\mathrm{e}}$ cure soit 3 mois après l'apparition du déficit moteur, justifiant la réalisation d'une ponction biopsie rénale (PBR). L'examen histopathologique de la PBR objectivait un rein de morphologie optique normale sans prolifération endo ou extracapillaire, ni épaississement des membranes basales glomérulaires. L'étude en immunofluorescence directe mettait en évidence des dépôts pariétaux extra-membraneux d'IgG et de C3. Ces dépôts granuleux étaient globaux et diffus. II n'était pas observé de dépôts immuns mésangiaux, ni au niveau des membranes basales tubulaires. Ces aspects histopathologiques étaient ceux d'une GEM débutante (stade 1) (fig. 1 et 2). L'antigénémie EBV par PCR qualitative en temps réel était positive à 3590 copies $/ \mathrm{ml}$ (seuil de charge virale élevée à 1000 copies par millilitre pour cette technique) plus de 6 mois après le début des symptômes.

La patiente bénéficiait alors de 3 séries d'injections de méthylprednisolone $\left(1 \mathrm{~g} / 1,73 \mathrm{~m}^{2} / \mathrm{j}\right.$ pendant $3 \mathrm{j}$ ) avec relais oral par prednisone. Au plan neurologique, la symptomatologie était lentement régressive sur 6 mois avec une récupération totale sur le plan moteur, une normalisation du fond d'œil et de l'EMG. Sur le plan néphrologique, la persistance d'une protéinurie significative justifiait le maintien d'une corticothérapie orale à faible dose et l'instauration d'un traitement par inhibiteur de l'enzyme de conversion (IEC) puis par ciclosporine $(3 \mathrm{mg} / \mathrm{kg}$ par jour). La symptomatologie glomérulaire régressait progressivement. Les taux résiduels (To) de ciclosporine avaient toujours été compris entre 99 et $147 \mathrm{ng} / \mathrm{ml}$. Les IEC ont pu être arrêtés après 6 mois de traitement. La patiente était toujours sous ciclosporine 16 mois après le début de ce traitement, soit 22 mois après le diagnostic de l'atteinte rénale. Elle présentait une protéinurie minime inférieure à $5 \mathrm{mg} / \mathrm{kg}$ toutes les $24 \mathrm{~h}$.

En synthèse, cette jeune fille de 12 ans a présenté, au décours d'une primo-infection atypique à EBV, une PRNA avec diplégie faciale et HTIC suivie d'une GEM. La symptomatologie neurologique était lentement régressive après 3 cures d'immunoglobulines intraveineuses puis 3 cures de corticoïdes intraveineux avec relais per os. La persistance d'une charge virale élevée plus de 6 mois après le début des symptômes était en faveur d'une infection chronique à EBV, bien que le traitement immunosuppresseur au long cours puisse en être la cause; l'atteinte rénale semblait quant à elle évoluer pour son propre compte et était stabilisée à 22 mois d'évolution sous ciclosporine et corticothérapie orale à faible dose.

\section{Discussion}

La PRNA ou syndrome de Guillain-Barré reste une maladie rare dont la prévalence est estimée entre 0,5 et 1,5 sur 100 ooo enfants dans les pays industrialisés [1]. Elle est la première cause de paralysie aiguë et extensive non traumatique depuis la disparition de la poliomyélite. Sa gravité est variable, et une ventilation mécanique est nécessaire dans 4 à $15 \%$ des cas pédiatriques. Quatre-vingt-dix à $95 \%$ des enfants bénéficient d'une récupération complète sans séquelle à un an, la plupart des autres conservant une déambulation autonome avec de minimes séquelles neurologiques [3]. Le traitement repose sur l'administration précoce de traitements immunomodulateurs comme les immunoglobulines intraveineuses (dose totale $2 \mathrm{~g} / \mathrm{kg}$ sur 2 à $4 \mathrm{j}$ ) ou les échanges plasmatiques 
(volume cumulé $250 \mathrm{ml} / \mathrm{kg}$ ) associés au traitement symptomatique [3].

Dans $2 / 3$ des cas de PRNA, on retrouve à l'anamnèse une infection dans les $30 \mathrm{j}$ précédant l'atteinte neurologique, avec des symptômes respiratoires ou grippaux dans 48 à $52 \%$ des cas et des symptômes digestifs dans $27 \%$ des cas [2]. SivadonTardy et al. ont étudié le profil sérologique de 263 cas de PRNA entre 1996 et 2001 en région parisienne [4] : Campylobacter jejuni était impliqué dans $21,9 \%$ des cas, le CMV dans $15,1 \%$, Mycoplasma pneumoniae dans 2,3\% et l'EBV dans 1,15\% des cas. Ces résultats étaient sensiblement similaires à ceux des études anglo-saxonnes [5].

Concernant l'EBV, il existe peu de données de forte puissance du fait que l'infection est quasi ubiquitaire et que les critères virologiques pour affirmer la primo-infection sont stricts (positivité des IgM anti-VCA et absence des anticorps antiEBNA). La première observation date de 1947 [6], des observations isolées ainsi que des séries ont été publiées, suggérant une association authentique. Finalement, l'EBV serait responsable de 1 à $8 \%$ des PRNA [4,5]. Le mécanisme physiopathologique de l'atteinte neurologique a été essentiellement étudié dans les PRNA secondaires aux infections à Campylobacter jejuni et $\mathrm{CMV}$ : un phénomène de mimétisme moléculaire serait responsable de la synthèse d'anticorps antigangliosides $[7,8]$. Une expression de CMV prolongée, la réactivation d'un virus latent ou une réinfection pourraient aussi intervenir [9]. Concernant les PRNA associées aux infections à EBV, la pathogénie est complètement inconnue mais, devant les similitudes entre EBV et CMV (tous les 2 appartenant au groupe des virus herpes), on est tenté d'évoquer des processus relativement similaires. L'association entre PRNA et glomérulopathie a été décrite dès 1918, soit 2 ans après la description originelle de la maladie par Guillain, Barré et Strohl [10], et elle n'est pas rare. On retrouve dans la littérature une nette prévalence de GEM, quelques atteintes à type de lésions glomérulaires minimes, hyalinoses segmentaires et focales, ou glomérulonéphrites (GN) post-infectieuses, et d'exceptionnelles GN membranoprolifératives [11-13]. L'atteinte glomérulaire ne s'accompagne pas nécessairement de syndrome néphrotique et dans un certain nombre de cas, on observe une protéinurie non néphrotique (c'est-à-dire inférieure à $50 \mathrm{mg} / \mathrm{kg}$ par jour) avec rémission rapide de l'atteinte rénale lors de la régression de la symptomatologie neurologique. On peut ainsi émettre l'hypothèse d'une prévalence de l'atteinte rénale au cours des PRNA plus élevée que celle communément admise, le diagnostic n'étant pas réalisé du fait de l'absence de dépistage et de la rémission rapide de la protéinurie. La chronologie d'apparition de la glomérulopathie est variable, pouvant survenir lors de la phase d'installation de la PRNA, lors de la décroissance des symptômes (comme c'était le cas chez notre patiente) voire même à distance lorsque la récupération a été complète au plan neurologique. La pathogénie de l'association entre l'atteinte glomérulaire et l'atteinte neurologique reste spéculative et plusieurs hypothèses peuvent être évoquées : une accumulation de complexes anticorps-antigène neural dans le glomérule, un antigène exogène (par exemple viral) atteignant le nerf et le glomérule ou bien une atteinte spécifiquement auto-immune des 2 appareils rénal et neurologique [11].

L'infection à EBV, aiguë ou chronique, est associée à de multiples formes d'atteinte rénale, allant de l'hématurie microscopique à l'insuffisance rénale aiguë. Plusieurs types de glomérulopathies ont déjà été décrits dans le cadre d'infections aiguës comme chroniques, chez l'adulte et chez l'enfant : GEM [14], GN membranoproliférative, GN avec dépôts de complexes immuns (GN aiguë post-infectieuse), néphropathies à dépôts mésangiaux d'IgA. La réalisation de PCR EBV sur des biopsies rénales de patients porteurs de différentes néphropathies glomérulaires a montré que la présence du virus n'était pas spécifique de la maladie, et que l'EBV était plus fréquemment associé aux atteintes glomérulaires et mésangiale. La pathogénie de l'atteinte rénale est inconnue mais ferait probablement intervenir des complexes immuns [15].

L'observation que nous rapportons se caractérise par la sévérité du tableau clinique, qui était également particulier par sa complexité : déficit moteur mais aussi ataxie, HTIC et diplégie faciale. L'HTIC et la diplégie faciale sont relativement rares dans la PRNA, mais l'ataxie est bien décrite dans un certain nombre de variants de la PRNA (notamment le syndrome de MillerFischer associant la triade ataxie-aréflexie-ophtalmoparésie). II est, en effet, probable que le retard à la prise en charge chez notre patiente (près d'un mois et demi après le début des difficultés motrices) soit en partie responsable de la mauvaise réponse initiale au traitement ayant justifié secondairement la réalisation de cures de corticoïdes intraveineux.

L'atteinte neurologique qu'avait présentée notre patiente semblait concomitante d'une primo-infection aiguë à EBV alors que l'atteinte glomérulaire s'était développée plus tardivement, vraisemblablement lors du passage à la chronicité de l'infection. Il est possible par ailleurs qu'une dysrégulation dans la réplication de l'EBV (comme le laisse suggérer la charge virale élevée dans le sang plus de 6 mois après la primo-infection) ait entraîné une synthèse excessive d'anticorps anti-EBNA puis une synthèse d'auto-anticorps, à l'image des réactions immunitaires croisées retrouvées dans les associations entre EBV et d'autres affections impliquant des processus auto-immuns (lupus érythémateux disséminé [16] et sclérose en plaques chez l'enfant [17], polyarthrite rhumatoïde [18]). L'infection virale semble ainsi avoir un rôle déclenchant dans le dérèglement de l'auto-immunité. Par ailleurs, cette charge virale élevée persistante peut en partie être expliquée par le traitement immunosuppresseur prescrit au long cours. Le bilan auto-immun est resté négatif chez notre patiente, mais le développement futur d'un connectivite avec atteinte rénale n'est pas à exclure.

Concernant le traitement immunosuppresseur par inhibiteur de la calcineurine (ciclosporine), une méta-analyse a conclu à 
l'efficacité de la ciclopsorine dans la prévention des rechutes des syndromes néphrotiques cortico-résistants de l'enfant, sans que la supériorité de ce traitement par rapport à d'autres immunosuppresseurs n'ait été prouvée [19]. La durée de traitement n'est pas consensuelle et peut dépasser 12 mois selon les études. De même, l'association de cette molécule à la corticothérapie au long cours, bien que pratiquée par de nombreuses équipes, n'a pas prouvé d'efficacité supérieure à un traitement par ciclosporine seule en cas de syndrome néphrotique idiopathique corticorésistant [20].

\section{Conclusion}

La PRNA est une urgence thérapeutique. Son association avec la primo-infection à EBV est bien décrite, bien que la pathogénie en soit inconnue. Une atteinte glomérulaire peut être associée à l'atteinte neurologique et a par ailleurs été décrite au cours de certaines infections à EBV indépendamment de toute polyradiculonévrite. Dans l'observation que nous rapportons, la maladie neurologique a été contrôlée en 6 mois malgré un retard au diagnostic, mais la glomérulopathie évolue désormais pour son propre compte. Le pronostic à long terme est devenu exclusivement rénal. Un bilan néphrologique semble ainsi nécessaire en cas de PRNA, les modalités exactes du suivi rénal à long terme chez ces patients restent à préciser. Nous n'avons pas retrouvé d'observation associant les 3 conditions (PRNA, GEM et primo-infection à EBV) dans la littérature française et anglo-saxonne.

\section{Conflit d'intérêt}

Aucun.

\section{Références}

[1] Olivé JM, Castillo C, Castro RG, et al. Epidemiologic study of Guillain-Barre syndrome in children $<15$ years of age in Latin America. J Infect Dis 1997;175 Suppl. 1:160-4.

[2] Koga M, Yuki N, Hirata K. Antecedent symptoms in GuillainBarre syndrome: an important indicator for clinical and serological subgroups. Acta Neurol Scand 2001;103:278-87.

[3] Sladky JT. Guillain-Barré syndrome in children. J Child Neurol 2004;19:191-200.
[4] Sivadon-Tardy V, Orlikowski D, Rozenberg F, et al. GuillainBarre syndrome, greater Paris area. Emerg Infect Dis 2006;12: 990-3.

[5] Hadden RD, Karch H, Hartung HP, et al. Preceding infections, immune factors, and outcome in Guillain-Barre syndrome. Neurology 2001;56:758-65.

[6] Ricker W, Blumberg A, Peters $\mathrm{CH}$, et al. The association of the Guillain-Barré syndrome with infectious mononucleosis with a report of two fatal cases. Blood 1947;2:217-22.

[7] Yuki N. Infectious origins of, and molecular mimicry in, Guillain-Barré and Fisher syndromes. Lancet Infect Dis 2001;1:29-37.

[8] Ang CW, Jacobs BC, Brandenburg AH, et al. Cross-reactive antibodies against $G M 2$ and $C M V$-infected fibroblasts in Guillain-Barré syndrome. Neurology 2000;54:1453-8.

[9] Steininger C, Seiser A, Gueler N, et al. Primary cytomegalovirus infection in patients with Guillain-Barre syndrome. J Neuroimmunol 2007;183:214-9.

[10] Bradford JR, Bashford EF, Wilson JA. Acute infectious polyneuritis. Q J Med 1918;12:88-126.

[11] Olbricht CJ, Stark E, Helmchen. et al. Glomerulonephritis associated with inflammatory demyelinating polyradiculoneuropathy: a case report and review of the literature. Nephron 1993;64:139-41.

[12] Kitamura H, Nakano T, Kakihara $M$, et al. A case of GuillainBarre syndrome developed minimal change nephrotic syndrome simultaneously. Am J Nephrol 1998;18:151-4.

[13] Emsley HC, Molloy J. Inflammatory demyelinating polyradiculoneuropathy associated with membranous glomerulonephritis and thrombocytopaenia. Clin Neurol Neurosurg 2002;105: 23-6.

[14] Araya CE, Gonzalez-Peralta RP, Skoda-Smith S, et al. Systemic Epstein-Barr virus infction associated with membranous nephropathy in children. Clin Nephrol 2006;65:160-4.

[15] Iwama H, Horikoshi S, Shirato I, et al. Epstein-Barr virus detection in kidney biopsy specimens correlates with glomerular mesangial injury. Am J Kidney Dis 1998;32:785-93.

[16] James JA, Kaufman KM, Farris AD, et al. An increased prevalence of Epstein-Barr virus infection in young patients suggests a possible etiology for systemic lupus erythematosus. J Clin Invest 1997;100:3019-26.

[17] Banwell B, Krupp L, Kennedy J, et al. Clinical features and viral serologies in children with multiple sclerosis: a multinational observational study. Lancet Neurol 2007;6:773-81.

[18] Toussirot E, Roudier J. Pathophysiological links between rheumatoid arthritis and the Epstein-Barr virus: an update. Joint Bone Spine 2007;74:418-26.

[19] Hodson EM, Willis NS, Craig JC. .Non-corticosteroid treatment for nephrotic syndrome in children. Cochrane Database Syst Rev 2008;23:CDoo2290.

[20] Hodson EM, Habashy D, Craig JC. Interventions for idiopathic steroid-resistant nephrotic syndrome in children. Cochrane Database Syst Rev 2006;19:CDo03594. 